\title{
Treatment of Refractory Rosacea with a Dual Wavelength Long- Pulsed 755-nm Alexandrite and 1,064-nm Nd:YAG Laser
}

Yoon Jin $\mathrm{Choi}^{1}$

Dae Suk Kim²

Suhyun $\mathrm{Cho}^{1}$

${ }^{1}$ Yonsei BB Skin Clinic, Seoul, Korea
${ }^{2}$ Yonsei Young Skin Clinic, Seoul, Korea

Received June 23, 2018

Accepted June 25, 2018

\section{Correspondence}

Suhyun Cho

Yonsei BB Skin Clinic, 551 Siheung-daero, Gurogu, Seoul 08392, Korea

Tel.: +82-2-837-8275

Fax: +82-2-837-8276

E-mail: lakeofwooddgmail.com

(C) Korean Society for Laser Medicine and Surgery

(c) This is an open access article distributed under the terms of the Creative Commons Attribution NonCommercial License (http://creativecommons.org/ licenses/by-nc/4.0) which permits unrestricted noncommercial use, distribution, and reproduction in any medium, provided the original work is properly cited.
The 755-nm and 1,064-nm lasers, when compared with the 595-nm pulsed dye laser, can provide deeper penetration into the dermis, allowing for targeting of deeper and larger vessels. Photon absorption by hemoglobin at 755- $\mathrm{nm}$ is better than that at 1,064-nm; hence, more efficient vascular ablation can be induced when using 755-nm laser. However, 755-nm laser is highly absorbed by melanin, and it should be used in caution considering epidermal damage and threshold fluences for vascular clearance. Microsecond Nd:YAG laser is less painful with good safety profiles compared with the traditional millisecond Nd:YAG laser and pulsed dye laser. Accordingly, the dual wavelength long-pulsed 755-nm alexandrite/microsecond 1,064-nm Nd:YAG laser, together with has both advantages of each laser and can show benefits with complementary, synergistic effects in treating rosacea. Herein, we describe two patients with rosacea who were effectively treated with the dual wavelength long-pulsed 755-nm alexandrite/microsecond 1,064-nm $\mathrm{Nd}: Y A G$ laser without noticeable side effects.

\section{Key words}

Rosacea; Dual wavelength; Long-pulsed; Alexandrite laser; Nd:YAG laser 


\section{INTRODUCTION}

Rosacea is a chronic, inflammatory disease which manifests with facial erythema, recurrent flushing, telangiectasia, persistent edema, and papulopustules. ${ }^{1,2}$ It mostly affects the central face, including the cheeks, nose, chin, and forehead. ${ }^{2}$ It is known to be more common in women and the symptoms usually starts during the middle age. ${ }^{3}$ The disease is characterized by its chronicity and recurrent exacerbation. ${ }^{3}$ Since the disease generally affects the face, it can lower the patient's self-esteem, cause stress and depression which can hinder the patient's quality of life. $^{4}$

Various treatment options are being used to treat rosacea, including medications such as topical and systemic antibiotics, isotretinoin, topical azelaic acid, tacrolimus, tranexamic acid, brimonidine tartrate, ivermectin, and lasers such as pulsed dye laser (PDL), long pulsed Nd:YAG laser (LPND), and intense pulsed light., ${ }^{5,6}$ Although these treatment options are all relatively effective in controlling rosacea, there are limitations with the effects of various therapies on remission rates and randomized clinical trial studies are insufficient in most modalities. ${ }^{1}$

Among laser devices, PDL has been most commonly used in treating facial erythema and telangiectasia as it is highly efficient in treating vascular lesions. ${ }^{5}$ However, there are quite a few cases which are refractory to PDL treatment, and various laser devices are being attempted in treating rosacea including the microsecond $\mathrm{Nd}: \mathrm{YAG}$ laser, dual-frequency ultrasound, radiofrequency devices, and dual wavelength long-pulsed 755-nm alexandrite/ microsecond 1,064-nm Nd:YAG laser., ${ }^{5,7-9}$ The microsecond $\mathrm{Nd}: Y A G$ laser is less painful and is less risky than the traditional millisecond LPND with a similar safety profile to that of PDL, and since alexandrite laser has a deeper penetration depth compared to PDL, it can also be effective in treating vascular lesions refractory to PDL. ${ }^{7.10}$ Therefore, the dual wavelength long-pulsed 755-nm alexandrite/microsecond 1,064-nm Nd:YAG laser can be a good selective option for rosacea treatment. In this report, we present cases of two patients with refractory rosacea effectively treated with the dual wavelength long-pulsed 755-nm alexandrite/microsecond 1,064-nm Nd:YAG laser.

\section{CASE 1}

A 50-year-old Korean male patient visited our clinic presenting with diffuse erythema on the face. He was suffering from recurrent flushing of the face for more than 3 years. He presented with widespread erythema and telangiectasia on the forehead, periorbital area, cheeks, and nose (Fig. 1A). According to his symptoms and clinical findings, he was diagnosed as erythematotelangiectatic type of rosacea. He did not have any pertinent family history or medical history and it was his first time visiting a dermatology clinic to consult for treatment options for his facial symptoms. He had never been treated with light or laser devices, oral or topical antibiotics, retinoids, or topical brimonidine tartrate gels formerly.

After obtaining a written informed consent, the patient's face was gently cleansed and laser treatment was performed without any topical anesthesia. The patient was treated with the dual wavelength long-pulsed 755-nm alexandrite/microsecond 1,064-nm Nd:YAG laser (Clarity ${ }^{\circledR}$; Lutronic Corp., Goyang, Koreal first using 755-nm

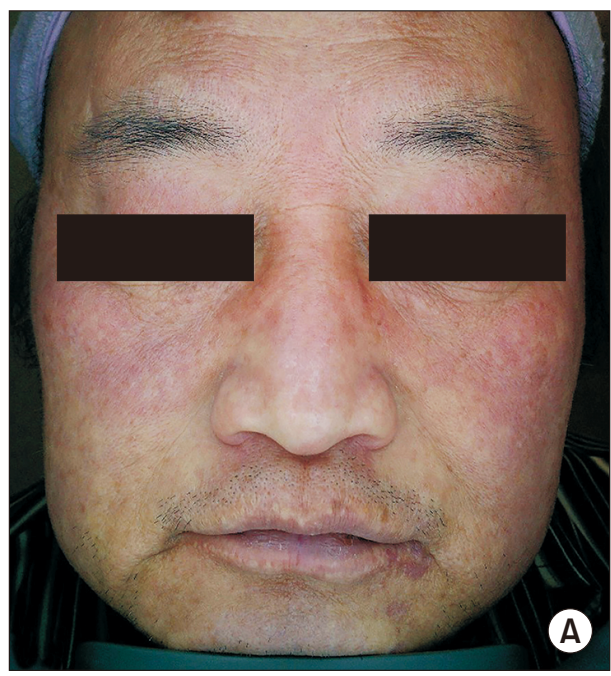

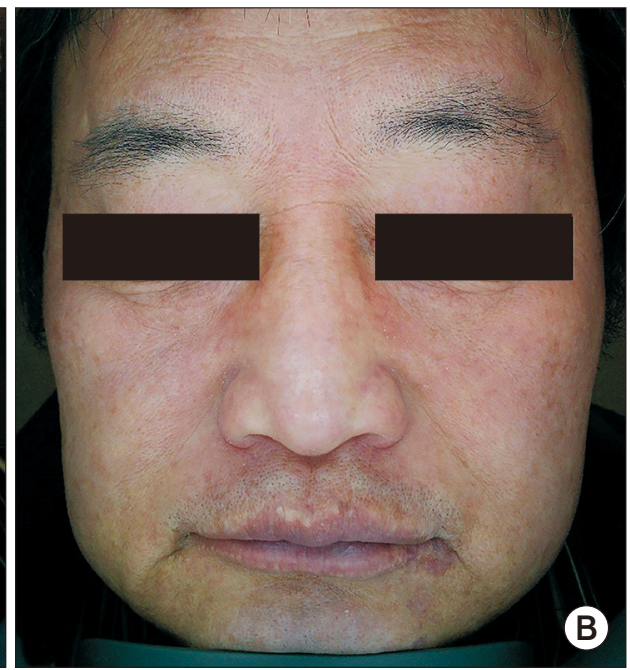

B
Fig. 1. Clinical photographs showing a 50-year-old Korean male with erythematotelangiectatic rosacea, (A) before and (B) 4 weeks after 6 sessions of dual wavelength longpulsed 755-nm alexandrite/microsecond 1,064-nm Nd:YAG laser. 
alexandrite laser with the fluence of $15 \mathrm{~J} / \mathrm{cm}^{2}$ with a spot size of $8 \mathrm{~mm}$ and pulse width of $5 \mathrm{~ms}$ for a single pass followed by a subsequent treatment with the fluence of 15 $\mathrm{J} / \mathrm{cm}^{2}$ with a spot size of $8 \mathrm{~mm}$ and pulse width of $12 \mathrm{~ms}$ for another pass. Then he was continuously treated with 1,064-nm Nd:YAG laser with the fluence of $20 \mathrm{~J} / \mathrm{cm}^{2}$ with a spot size of $8 \mathrm{~mm}$ and pulse width of $20 \mathrm{~ms}$ for 1 pass followed by another pass with the fluence of $30 \mathrm{~J} / \mathrm{cm}^{2}$ with a spot size of $8 \mathrm{~mm}$ and pulse width of $30 \mathrm{~ms}$. All treatments were tolerable without topical anesthesia and six treatment sessions were performed weekly. Immediately after the treatment, the patient's face showed only slight erythema and was cooled with icepacks. No systemic or topical antibiotics and/or corticosteroids were prescribed during the course of treatment.

After six sessions of dual wavelength long-pulsed 755$\mathrm{nm}$ alexandrite/microsecond 1,064-nm Nd:YAG laser treatment, the patient showed remarkable improvement in facial erythema and telangiectasia (Fig. 1B). Flushing symptoms were not experienced after 4 sessions of treatment. He was satisfied with the treatment results, and no remarkable side effects or discomfort, including crusting, bruising, bleeding, oozing, exacerbation of rosacea symptoms, post-laser prolonged erythema, or scarring were reported.

\section{CASE 2}

A 57-year-old Korean female patient visited our clinic presenting with persistent facial erythema and papulopustules on the cheeks, nose and perioral area (Fig. 2A). She was diagnosed as papulopustular rosacea. She had suffered from these facial symptoms for about 5 years and she had been intermittently treated with oral steroid and oral/topical antibiotics at another clinic for 3 years. However, she experienced recurrent exacerbation and she gave up treatment for the past 2 years. She had not been treated with light or laser devices, oral or topical antibiotics, retinoids, topical brimonidine tartrate gels, or topical ivermectin cream for the treatment of rosacea within the recent 2 years. She did not have any pertinent family history or medical history.

After obtaining a written informed consent, the patient's face was gently cleansed and laser treatment was performed without topical anesthesia. The patient was treated with the dual wavelength long-pulsed 755-nm alexandrite/microsecond 1,064-nm Nd:YAG laser (Clarity ${ }^{\circledR}$ ) first using $755-\mathrm{nm}$ alexandrite laser with the fluence of $14 \mathrm{~J} / \mathrm{cm}^{2}$ with a spot size of $8 \mathrm{~mm}$ and pulse width of 5 ms for a single pass followed by a subsequent treatment with the fluence of $14 \mathrm{~J} / \mathrm{cm}^{2}$ with a spot size of $8 \mathrm{~mm}$ and pulse width of $12 \mathrm{~ms}$ for another pass. Then she was continuously treated with 1,064-nm Nd:YAG laser with the fluence of $20 \mathrm{~J} / \mathrm{cm}^{2}$ with a spot size of $8 \mathrm{~mm}$ and pulse width of $20 \mathrm{~ms}$ for 1 pass. Six treatment sessions were performed weekly, and all treatments were tolerable without topical anesthesia. Immediately after the treatment, the patient's face showed only slight erythema and was cooled with icepacks. No systemic or topical antibiotics and/or corticosteroids were prescribed during the course of treatment.

After six sessions of dual wavelength long-pulsed 755$\mathrm{nm}$ alexandrite/microsecond 1,064-nm Nd:YAG laser treatment, the patient showed marked improvement in facial erythema and the papulopustules (Fig. 1B). She was satisfied with the results, and no remarkable side effects



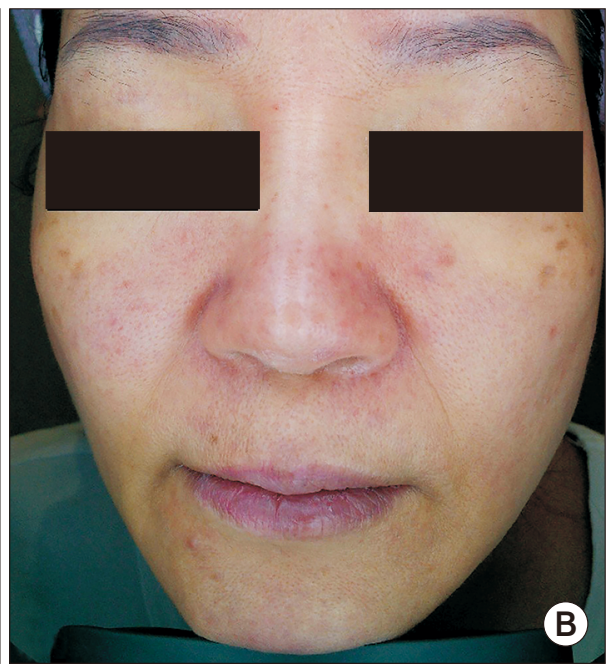

B
Fig. 2. Clinical photographs showing a 57-year-old Korean female with papulopustular rosacea, (A) before and (B) 4 weeks after 6 sessions of dual wavelength longpulsed 755-nm alexandrite/microsecond 1,064-nm Nd:YAG laser. 
or discomfort, including crusting, bruising, bleeding, oozing, exacerbation of rosacea symptoms, post-laser prolonged erythema, or scarring were reported.

\section{DISCUSSION}

Alexandrite laser has a deeper penetration depth compared to PDL due to its longer wavelength. This makes it possible to target larger and deeper vasculature. ${ }^{5,10}$ LPND has been effectively used to treat cutaneous vascular lesions, and it has also been reported as a safe, effective treatment modality for the inflammatory and vascular lesions of rosacea. ${ }^{11}$ In a double-blind, randomized controlled study comparing the effect of microsecond $\mathrm{Nd}$ :YAG laser and PDL in treating diffuse facial erythema, nonpurpuric PDL turned out to be more effective, but the microsecond Nd:YAG was less painful. ${ }^{7}$ Therefore, the microsecond Nd:YAG laser can be a safe and less painful treatment option for rosacea. The dual wavelength long-pulsed 755-nm alexandrite/microsecond 1,064-nm $\mathrm{Nd}$ :YAG laser, thus can have a synergistic effect, each assisting the efficacy of the other wavelength laser on treating rosacea.

Photon absorption by hemoglobin at 755-nm is twice of it as at 1,064-nm. ${ }^{10}$ Accordingly, vascular heating can be more effectively induced when using 755-nm laser compared to 1,064-nm. However, alexandrite laser has a high melanin:hemoglobin absorption coefficient ratio than 595$\mathrm{nm}$ and 1,064-nm lasers, and this high melanin absorption should always be considered when treating vasculature using alexandrite laser. ${ }^{10,12}$ Treatment parameters should be adjusted considering to the patient's skin type and existence of epidermal/dermal pigmentations.

According to the modified National Rosacea Society classification system, rosacea has been classified into 4 subtypes and 1 variant, which are erythematotelangiectatic, papulopustular, phymatous, ocular, and granulomatous, respectively. ${ }^{2}$ Treatment of rosacea should be regulated considering the characteristics of each subtype. Although the exact pathophysiology of rosacea is still unclear, various factors such as vascular dysfunction and dilatation, release of inflammatory mediators, and microorganisms are known to be involved. 'Laser devices can be used to affect various factors which contribute to the pathophysiology of rosacea including vascular ablation, reducing inflammation and the release of inflammatory mediators, regeneration and remodeling of the dermal connective tissues. ${ }^{11,13}$

The long-pulsed alexandrite laser and Nd:YAG laser can penetrate deeper into the dermis compared to PDL, and therefore deeper and larger tissues including vessels can be targeted and ablated. ${ }^{5}$ These longer wavelength lasers can also penetrate more effectively into the dilated capillary walls. ${ }^{14}$ LPND has been reported to be effective in both treating the vascular lesions and inflammatory papulopustules of rosacea. ${ }^{11}$ LPND is also effective for dermal rejuvenation, which can be induced by the remodeling of dermal connective tissues. ${ }^{15}$ Therefore, as a dual wavelength laser, the long-pulsed 755-nm alexandrite/ microsecond 1,064-nm Nd:YAG laser can have synergistic effect strengthening each other's advantages in treating rosacea. It has been reported in the literature that there was no significant difference between the treatment results using dual wavelength long-pulsed 755-nm alexandrite/microsecond 1,064-nm Nd:YAG laser and PDL in rosacea patients. ${ }^{5}$

In this report, we demonstrated two patients with different subtypes of rosacea who were successfully treated with a dual wavelength long-pulsed 755-nm alexandrite/ microsecond 1,064-nm Nd:YAG laser. Remarkable improvements of background erythema, telangiectasia, flushing, and inflammatory lesions were noticed after six sessions of treatments. No purpura, bruising, bullae or any other side effects were observed. In conclusion, dual wavelength long-pulsed 755-nm alexandrite/microsecond 1,064-nm Nd:YAG laser turned out to be safe and effective with marked clinical outcomes in the treating rosacea. However, this study has some limitations that the maintenance duration of the effect of laser treatment was not observed for a long time period. Further evaluation with long term follow-up studies and with larger group of patients are needed to further confirm the effects.

\section{REFERENCES}

1. van Zuuren EJ. Rosacea. N Engl J Med 2017;377:1754-64.

2. Wilkin J, Dahl M, Detmar M, Drake L, Feinstein A, Odom R, et al. Standard classification of rosacea: report of the National Rosacea Society Expert Committee on the classification and staging of Rosacea. J Am Acad Dermatol 2002;46:584-7.

3. Elewski BE, Draelos Z, Dréno B, Jansen T, Layton A, Picardo M. Rosacea - global diversity and optimized outcome: proposed international consensus from the Rosacea International Expert Group. J Eur Acad Dermatol Venereol 2011;25:188-200.

4. Aksoy B, Altaykan-Hapa A, Egemen D, Karagöz F, Atakan N. The impact of rosacea on quality of life: effects of demographic and clinical characteristics and various treatment modalities. Br J Dermatol 2010;163:719-25.

5. Seo HM, Kim JI, Kim HS, Choi YJ, Kim WS. Prospective comparison of dual wavelength long-pulsed 755-nm 
Alexandrite/1,064-nm neodymium:yttrium-aluminum-garnet laser versus 585-nm pulsed dye laser treatment for rosacea. Ann Dermatol 2016;28:607-14.

6. Abokwidir M, Feldman SR. Rosacea management. Skin Appendage Disord 2016;2:26-34.

7. Alam M, Voravutinon N, Warycha M, Whiting D, Nodzenski M, Yoo S, et al. Comparative effectiveness of nonpurpuragenic 595-nm pulsed dye laser and microsecond 1064-nm neodymium:yttrium-aluminum-garnet laser for treatment of diffuse facial erythema: a double-blind randomized controlled trial. J Am Acad Dermatol 2013;69:438-43.

8. Park JY, Ahn MK, Cho EB, Park EJ, Kim KH. Dual-frequency ultrasound as a new treatment modality for refractory rosacea: a retrospective study. Dermatol Surg. In press 2018.

9. Ahn TH, Cho SB. Invasive pulsed-type, bipolar, alternating current radiofrequency treatment using microneedle electrodes for nasal rosacea. Med Lasers 2017;6:32-6.

10. Ross EV, Meehan KJ, Domankevitz Y, Trafeli JP, Annandono J, Jacoby M. Use of a variable long-pulse alexandrite laser in the treatment of facial telangiectasia. Dermatol Surg 2010;36:4704.

11. Say EM, Okan G, Gökdemir G. Treatment outcomes of longpulsed $\mathrm{Nd}$ : YAG laser for two different subtypes of rosacea. J Clin Aesthet Dermatol 2015;8:16-20.

12. Roggan A, Friebel M, Do Rschel K, Hahn A, Mu Ller G. Optical properties of circulating human blood in the wavelength range 400-2500 nm. J Biomed Opt 1999;4:36-46.

13. Pelle MT, Crawford GH, James WD. Rosacea: II. Therapy. J Am Acad Dermatol 2004;51:499-512.

14. Li L, Kono T, Groff WF, Chan HH, Kitazawa Y, Nozaki M. Comparison study of a long-pulse pulsed dye laser and a long-pulse pulsed alexandrite laser in the treatment of port wine stains. J Cosmet Laser Ther 2008;10:12-5.

15. Lee YB, Shin JY, Cheon MS, Oh ST, Cho BK, Park HJ. Photorejuvenation using long-pulsed alexandrite and long-pulsed neodymium:yttrium-aluminum-garnet lasers: a pilot study of clinical outcome and patients' satisfaction in Koreans. J Dermatol 2012;39:425-9. 